\title{
Use of fluorescence induction and sucrose counterselection to identify Mycobacterium tuberculosis genes expressed within host cells
}

\author{
James A. Triccas, François-Xavier Berthet, $\uparrow$ Vladimir Pelicicł \\ and Brigitte Gicquel
}

Unité de Génétique

Mycobactérienne, Institut

Pasteur, 25 rue du Dr Roux

75724 Paris Cedex 15, France
Author for correspondence: Brigitte Gicquel. Tel: +33 1456888 28. Fax: +3314568 8843 . e-mail: bgicquel@pasteur.fr

The identification of Mycobacterium tuberculosis genes expressed within host cells would contribute greatly to the development of new strategies to combat tuberculosis. By combining the natural fluorescence of the Aequoria victoria green fluorescent protein (GFP) with the counterselectable property of the Bacillus subtilis SacB protein, $M$. tuberculosis promoters displaying enhanced in vivo activity have been isolated. Macrophages were infected with recombinant Mycobacterium bovis bacille Calmette-Guérin containing a library of $M$. tuberculosis promoters controlling gfp and sacB expression, and fluorescent bacteria recovered by fluorescence-activated cell sorting. The expression of $\operatorname{sacB}$ was used to eliminate clones with strong promoter activity outside the macrophage, resulting in the isolation of seven clones containing $M$. tuberculosis promoters with greater activity intracellularly. The gene products identified displayed similarity to proteins from other organisms whose functions include nutrient utilization, protection from oxidative stress and defence against xenobiotics. These proposed functions are consistent with conditions encountered within the host cell and thus suggest that the augmented activity of the isolated promoters/genes may represent strategies employed by $M$. tuberculosis to enhance intracellular survival and promote infection.

Keywords: Mycobacterium tuberculosis, macrophage, gene induction, fluorescence

\section{INTRODUCTION}

Tuberculosis remains a significant global public health problem, representing a major cause of morbidity and mortality worldwide (Raviglione et al., 1995). Mycobacterium tuberculosis, the causative agent of tuberculosis, is a facultative intracellular pathogen with the ability to survive and replicate within host cells, in particular those of the mononuclear phagocytic lineage. Therefore, the development of new strategies to combat tuberculosis will in part rely on an understanding of the mechanisms employed by the bacterium to enhance

\footnotetext{
† Present address: SmithKline Beecham Biologicals, Laboratory of Molecular Bacteriology, Rue de I'Institut 89, B-1330 Rixensart, Belgium. $¥$ Present address: INSERM U411, Faculté de Médecine Necker-Enfants Malades, 156, Rue de Vaugirard, 75730 Paris Cedex 15, France.

Abbreviations: GFP, green fluorescent protein; (r)BCG, (recombinant) bacille Calmette-Guérin; FACS, fluorescence-activated cell sorting.
}

intracellular survival. These mechanisms are complex and varied, ranging from alterations in the characteristics of the macrophage phagosome (the compartment in which $M$. tuberculosis resides) to promote persistence (Clemens, 1996) to modification of the antigen-presenting ability of infected macrophages, contributing to immune evasion (Gercken et al., 1994). Despite the complex array of methods M. tuberculosis possesses to promote intracellular persistence, the molecular mechanisms by which M. tuberculosis resists host anti-bacterial functions remain to be elucidated.

The results of previous studies with other intracellular bacteria, such as Salmonella and Listeria, indicate that the adaptation to an intracellular environment involves modification in the pattern of bacterial gene expression (Moors \& Portnoy, 1995). By analogy, one would assume that similar mechanisms are employed by $M$. tuberculosis and to this end the identification of those M. tuberculosis genes whose expression is preferentially 
up-regulated within host cells would contribute significantly to our understanding of the organism's pathogenicity. The application of the Aequoria victoria green fluorescent protein (GFP) as a selectable marker to analyse bacterial gene expression has enhanced the development of methods to identify in vivo induced genes, such as the strategy termed differential fluorescence induction (DFI) (Valdivia \& Falkow, 1996). This technique permits the assessment of promoter activity in different environmental conditions by the analysis of GFP fluorescence, with recombinant bacteria of interest capable of being separated by fluorescence-activated cell sorting (FACS). This system was initially applied to the isolation of $\mathrm{pH}$-regulated promoters of Salmonella typhimurium (Valdivia \& Falkow, 1996) and later extended to identify promoters of the same organism showing differential activity outside and within host cells (Valdivia \& Falkow, 1997). The observation that GFP is efficiently expressed in mycobacteria and that fluorescent bacteria can be directly observed in infected macrophages (Dhandayuthapani et al., 1995; Kremer et al., 1995) suggested that such a strategy may be applicable to the study of gene induction by mycobacteria in host cells. A similar system to DFI was recently used to identify promoters of Mycobacterium marinum which show elevated levels of expression within macrophages compared to the extracellular environment (Barker et al., 1998).

While GFP represents a useful tool for the study of mycobacterial genetics, another genetic system proving extremely advantageous is that based on the $s a c B$ gene of Bacillus subtilis. This gene encodes a secreted enzyme that confers sucrose sensitivity on mycobacteria (Pelicic et al., 1996a). This property has been used to efficiently perform allelic exchange and construct transposon mutant libraries in both the fast- and slow-growing mycobacteria (Pelicic et al., 1996b, 1997). In this study, we have combined the features of both the GFP and SacB proteins to develop a system permitting the isolation of genes of M. tuberculosis differentially expressed within host cells. Macrophages were infected with recombinant ( $\mathrm{r}$ ) Mycobacterium bovis bacille Calmette-Guérin (BCG) containing a library of $M$. tuberculosis promoters controlling $g f p$ and $s a c B$ expression, and GFP-positive bacteria sorted by FACS. SacB expression was then used to eliminate $\mathrm{rBCG}$ clones with strong promoter activity outside the macrophage, leading to the enrichment of clones containing M. tuberculosis promoters differentially expressed intracellularly.

\section{METHODS}

Bacterial strains and growth conditions. Escherichia coli $\mathrm{DH} 5 \alpha$ was grown routinely on liquid or solid Luria-Bertani medium. M. tuberculosis 103 (isolated directly from a tuberculosis patient; laboratory collection) and M. bovis BCG Pasteur were grown in liquid Middlebrook 7H9 medium (Difco) supplemented with ADC enrichment (Difco) or on solid Middlebrook 7H10 medium (Difco) supplemented with OADC enrichment (Difco). When required, kanamycin was added at a concentration of $25 \mu \mathrm{g} \mathrm{ml}^{-1}$ for both E. coli and mycobacteria. For the counterselection of sucrose-sensitive
M. bovis BCG, bacteria were grown in $7 \mathrm{H} 9$ medium supplemented with $0 \cdot 01-3 \%$ sucrose.

Vector construction. The promoterless B. subtilis $s a c B$ gene was amplified from the pJQ200 vector (Quandt \& Hynes, 1993) using the primers SacB1 (5'-GGGGCTGCAGAAAAAGGAGACATGAACGATGAACATC) and SacB2 (5'-GGGGCTGCAGTATGGGATTCACCTTTATGTTGATAAG). The PstI-digested product was cloned into PstI-digested pKEN-GFPmut2 (Cormack et al., 1996), placing the sacB gene directly downstream of a promoterless, highly fluorescent version of the Aequoria victoria $g f p$ gene to give plasmid pIPX39. We next constructed a suitable mycobacterial-E. coli shuttle vector for subsequent cloning procedures. The kanamycin resistance gene was cloned into $\mathrm{NdeI} / \mathrm{BsaI}$-digested pUC18, resulting in plasmid pPV8. The origin of replication of the M. fortuitum plasmid pAL5000 (Ranes et al., 1990) was then cloned into the StuI site of pPV8. The resulting plasmid, pPV24, is a multipurpose shuttle cloning vector harbouring five unique restriction sites (KpnI, BamHI, XbaI, PstI, SphI) and allows blue/white selection of recombinants in E. coli. The plasmid pJFX1 was constructed by digesting pIPX39 with BamHI and SphI and cloning of the resulting $2 \cdot 2 \mathrm{~kb}$ fragment into BamHI/SphI-digested pPV24. The tT4 transcription terminator was amplified from the vector pJEM12 with primers TT4.FOR (5'-AGACTAGATCTGTCATGCCATCCG) and TT4.REV (5'-TATCCGGATCCGCGGGCCCAG), digested with BamHI and BglII, and cloned into BamHIdigested $\mathrm{pJFX} 1$, giving $\mathrm{pJFX} 2$. The strong $\beta$-lactamase promoter of Mycobacterium fortuitum (Timm et al., 1994) was cloned into ScaI/BamHI-digested pJFX2 to give pJFX4.

Construction of the $M$. tuberculosis genome library. $M$. tuberculosis 103 DNA was extracted as previously described (Otal et al., 1991) and digested with Sau3A (0.03 U per $5 \mu \mathrm{g}$ ) for $20 \mathrm{~min}$. DNA was run on low-melting-temperature agarose (Bio-Rad), then the $200 \mathrm{bp}-1.5 \mathrm{~kb}$ fraction was purified (QIAquick gel extraction kit, Qiagen) and ligated to dephosphorylated, BamHI-digested pJFX2. DNA was extracted from approximately 10000 recombinant colonies and electroporated into M. bovis BCG.

Macrophage preparation and infection. Bone-marrow-derived macrophages were isolated from the femurs of 7-weekold female Balb/c mice. Cells were seeded at $2 \times 10^{5}$ macrophages per well in 24-well plates (Costar) and incubated at $37^{\circ} \mathrm{C}$ in $5 \% \mathrm{CO}_{2}$ for $7 \mathrm{~d}$. Macrophage monolayers were infected with bacteria at a m.o.i. of $1: 1$. After $4 \mathrm{~h}$ of infection, extracellular bacteria were removed by washing four times with PBS and incubation continued at $37^{\circ} \mathrm{C}$ in $5 \% \mathrm{CO}_{2}$.

FACS analysis and sorting of fluorescent macrophages. Bacteria and macrophages were analysed with a FACScan (Becton-Dickinson) and sorted with an ELITE cell sorting system (Beckman Coulter Inc.). After 5-6 d of infection, BCGinfected macrophages were washed three times in PBS, scraped into $1 \mathrm{ml}$ PBS and analysed by FACS. Macrophages exhibiting fluorescence levels greater than those infected with $M$. bovis BCG harbouring pJFX2 alone were collected. To recover bacteria, sorted macrophages were centrifuged and lysed in water plus $0 \cdot 1 \%$ Tween 80 . Recovered bacteria were grown in 7H9 medium for $7 \mathrm{~d}$ and macrophage infection and sorting repeated until the desired level of enrichment of macrophage/ fluorescent bacteria was attained.

Selection of clones with enhanced intracellular expression. Macrophages in 24-well culture plates were infected with recombinant BCG clones recovered after the selection process at a m.o.i. of $1: 1$. After $4 \mathrm{~h}$ of infection, macrophages were washed four times with PBS and the infection continued or 
bacteria recovered from Tween-80-lysed macrophages for FACS determination of fluorescence levels (representing day $0)$. After $6 \mathrm{~d}$ of infection, the remaining macrophages were washed four times with PBS, lysed and bacterial fluorescence levels determined by FACS. Clones displaying a day 6 to day 0 fluorescence ratio of approximately 2 or greater were considered to display significant intracellular induction. A total of 2000 bacteria from culture medium alone or lysed macrophage were analysed as a function of side scatter and GFP fluorescence. Quantification of fluorescence levels measured by FACS was determined by the use of the Lysis II program (Becton-Dickinson), while fluorescence of bacterial suspensions and colonies was visualized directly using a Axioscop fluorescence microscope (Zeiss).

PCR and sequencing of selected clones. DNA from clones of interest was extracted by freeze-thawing of bacterial suspensions and subjected to PCR amplification using primers BCGPR.FOR1 (5'-AACGGGATCCAAGCATAAAGCTAGT) and BCGPR.REV (5'-TTACGGATCCGTATATCTCCTTCTT). PCR fragments were sequenced using a model 373 automated DNA sequencer (Applied Biosystems). DNA homologies were determined using the M. tuberculosis BLAST server at the Sanger Centre (www.sanger.ac.uk).

\section{RESULTS}

\section{Construction and validation of the GFP-SacB- promoter selection vector}

To develop a system to enrich for promoters that show enhanced activity within host cells, we constructed the pJFX2 vector (Fig. 1). This E. coli/mycobacterial shuttle vector contains a promoterless copy of the B. subtilis $s a c B$ gene directly preceded by a promoterless copy of the gene encoding a mutated version of GFP (Cormack et al., 1996). This form of GFP exhibits greater fluorescence and solubility compared to the wild-type protein.

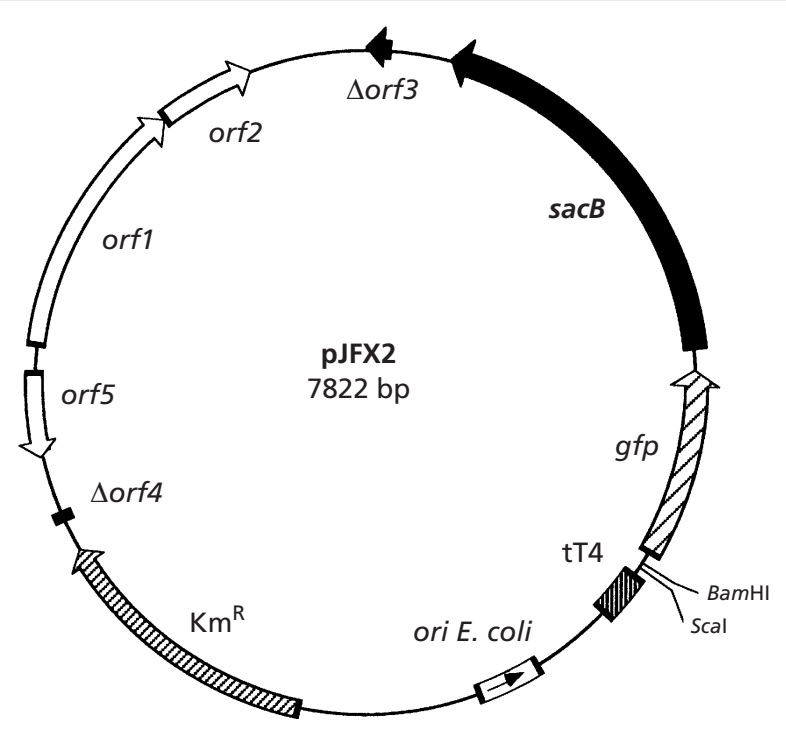

Fig. 1. Map of the pJFX2 vector used for the isolation of mycobacterial promoters induced in vivo. The unique Scal and $B a m H I$ sites used for cloning of promoter fragments directly upstream of the $g f p / s a c B$ operon are shown. tT4 represents the transcriptional terminator of coliphage T4.
A transcription terminator located upstream of the $g f p / s a c B$ operon ensures that $g f p / s a c B$ expression is by virtue of promoters placed before the operon.

To determine if GFP in this vector is functional, we placed the $g f p / s a c B$ operon in pJFX2 under the control of the strong $\beta$-lactamase promoter of $M$. fortuitum (Timm et al., 1994), resulting in pJFX4. M. bovis BCG harbouring pJFX4 exhibited strong fluorescence as observed by UV microscopy and FACS analysis (data not shown). By contrast, M. bovis BCG carrying pJFX2 alone was non-fluorescent.

The growth of $M$. bovis BCG harbouring pJFX2 on plates containing 0.5 and $5 \%$ sucrose was not affected, while $M$. bovis BCG carrying pJFX4 was killed in the presence of 0.5 and $5 \%$ sucrose (data not shown). This suggested that expression of the $s a c B$ gene of pJFX2 was sufficient to permit effective counterselection of rBCG. We next determined if such $s a c B$ expression could permit the regulated elimination of $s a c B$-expressing bacteria. A promoter library of $M$. tuberculosis 103 DNA $(0 \cdot 2-1 \cdot 5 \mathrm{~kb})$ was constructed in pJFX2 and transformed into M. bovis BCG, resulting in a pool of BCG clones exhibiting varying levels of GFP expression as assessed by FACS (see Fig. 3a).To obtain a population containing a sufficient proportion of $g f p / s a c B$-expressing bacteria to allow detailed analysis, GFP-positive $M$. bovis BCG from the pool of clones were sorted by FACS and the subsequent population re-analysed. After one round of sorting, the proportion of GFP-positive BCG rose from 9 to $41 \%$, with varying levels of fluorescence (Fig. 2a). As $s a c B$ and $g f p$ are present in an operon, then it can be assumed that a similar variety in $s a c B$ expression is present in this library. To test and monitor the regulation of counterselection by the use of sucrose, the effect of both sucrose concentration and incubation time on the killing of sacB-expressing bacteria was analysed. The pooled BCG clones were grown to an $\mathrm{OD}_{600}$ of 0.25 at which point sucrose, ranging in concentration from 0.01 to $3 \%$, was added and the proportion of GFP-positive bacteria analysed by FACS at varying time points. As seen in Fig. 2(b), while no or low concentrations of sucrose $(0 \cdot 1 \%)$ had a minimal effect on the proportion of GFP-positive bacteria observed, increasing the concentration of sucrose in the medium led to an increased elimination of GFP-positive bacteria, in a dose-dependent manner. Analysis of individual FACS profiles (Fig. 2a) suggested that it was those bacteria expressing high levels of $g f p / s a c B$ that were being preferentially eliminated by the effect of sucrose. To confirm this, we determined the mean fluorescence level of GFP-positive bacteria over time after the addition of $0.3 \%$ sucrose to the $M$. bovis BCG pool. As shown in Fig. 2(c), the mean fluorescence of the population steadily decreased with time, indicating that it was strong $g f p / s a c B$-expressing bacteria that were being eliminated initially, with longer periods of incubation required to eliminate bacteria expressing intermediate or low levels of $g f p / s a c B$. Taken together, these results suggest that varying the concentration of sucrose and time of incubation can be used to achieve 
(a)
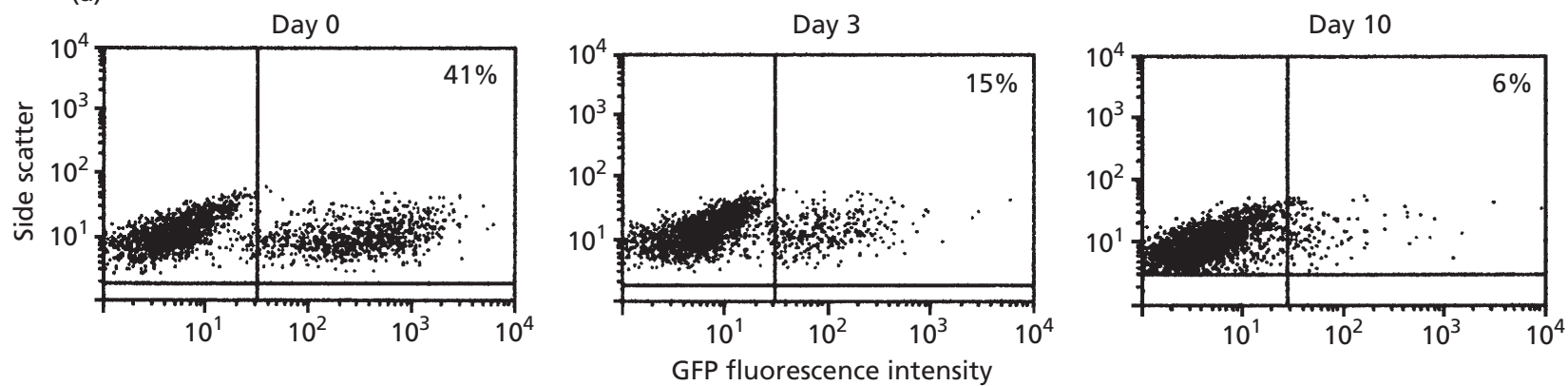

(b)

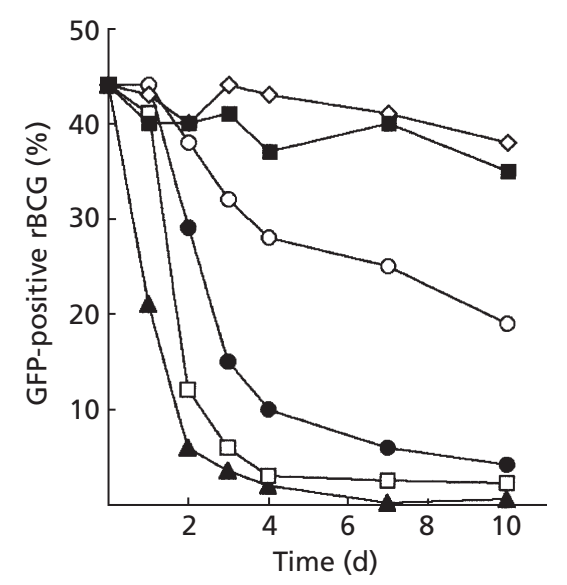

(c)

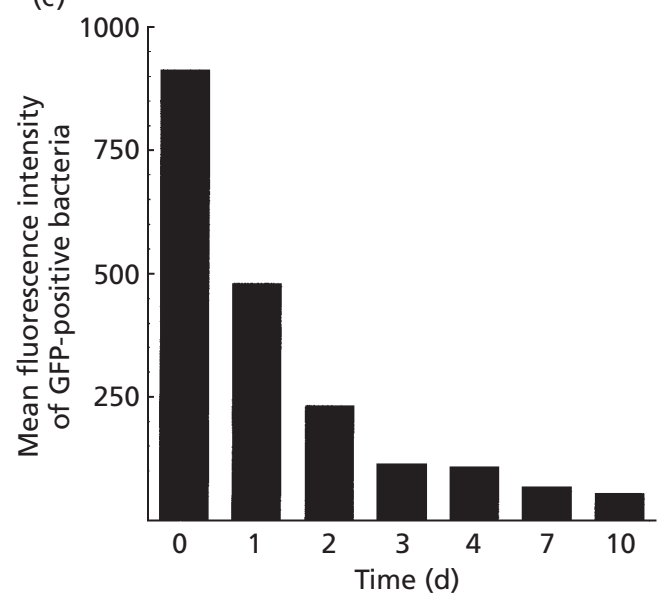

Fig. 2. Use of sucrose counterselection to permit the controlled elimination of $g f p / s a c B$ expressing rBCG. (a) Representative FACS plots of the change in the proportion over time of GFP-positive rBCG grown in 7 H9 growth medium supplemented with $0.3 \%$ sucrose. The percentage of GFP-positive bacteria present at each given time point is shown. (b) The effect of changes in sucrose concentration and incubation time on the proportion of GFP-positive rBCG harbouring random promoter fragments of $M$. tuberculosis. The rBCG pool was grown in 7H9 growth medium supplemented with varying sucrose concentrations $(\%: \mathbf{\square}, 0 ; \diamond, 0.03 ; \bigcirc, 0.1 ; 0,0.3 ; \square, 1 ; \boldsymbol{\Lambda}, 3)$ and the percentage of GFP-positive bacteria remaining at each given time point was determined by FACS analysis. (c) Changes in the mean fluorescence of GFPpositive rBCG over time due to sucrose counterselection. The mean fluorescence of only those GFP-positive bacteria remaining at each given time point after incubation with $0.3 \%$ sucrose was calculated by FACS analysis.

selective elimination of $s a c B$-expressing bacteria based on the strength of the promoter that they harbour.

\section{Selection of rBCG clones displaying enhanced fluorescence within host cells}

A strategy based on the fluorescence of GFP and the sensitive counterselective property of $\mathrm{SacB}$ was developed to allow the isolation of $M$. tuberculosis promoters displaying enhanced activity intracellularly. Macrophages were infected for $6 \mathrm{~d}$ with rBCG harbouring a $M$. tuberculosis DNA library in pJFX2 (Fig. 3a) and fluorescent macrophages were sorted by FACS. Macrophages were lysed, bacteria recovered and rested in culture medium for $7 \mathrm{~d}$, and macrophages were reinfected with this rBCG pool. This process was repeated three times, after which a population of rBCG enriched for fluorescent bacteria was obtained (Fig. 3b). We then used the expression of $s a c B$ to eliminate those bacteria which contained $M$. tuberculosis promoters that functioned strongly outside the macrophage. Based on the results displayed in Fig. 2, we surmised that incubation with $0.3 \%$ sucrose for $6 \mathrm{~d}$ would allow elimination of the majority of bacteria expressing $s a c B$ strongly ex vivo. The macrophage fluorescence-enriched pool of rBCG (Fig. 3b) was treated with $0.3 \%$ sucrose for $6 \mathrm{~d}$, which resulted in a pool of rBCG expressing no or low levels of $g f p / s a c B$ (Fig. 3c). This population was then used to reinfect macrophages for a final time $(6 \mathrm{~d})$, the macrophages were lysed and GFP-positive bacteria were sorted and plated onto solid medium. In parallel, we also isolated individual bacteria from the macrophage fluorescence-enriched pool of rBCG not treated with sucrose (Fig. 3b) to isolate promoters that may exhibit medium levels of extracellular activity and which are up-regulated during infection, yet would have been eliminated during the sucrose selection step.

Colonies obtained after growth on solid medium were cultured individually in 24-wells plates and used to infect macrophages for $6 \mathrm{~d}$. The level of fluorescence in 7H9 medium and in macrophages $4 \mathrm{~h}$ or $6 \mathrm{~d}$ after infection was compared by FACS. We considered 
(a)

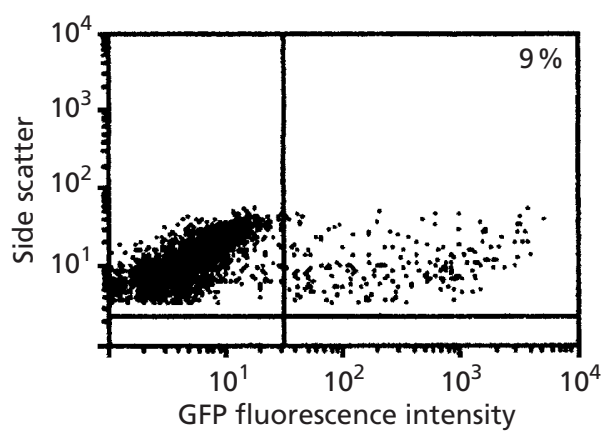

- Macrophage infection

- Enrichment of fluorescent

macrophages by FACS

- Recovery of bacteria (b)
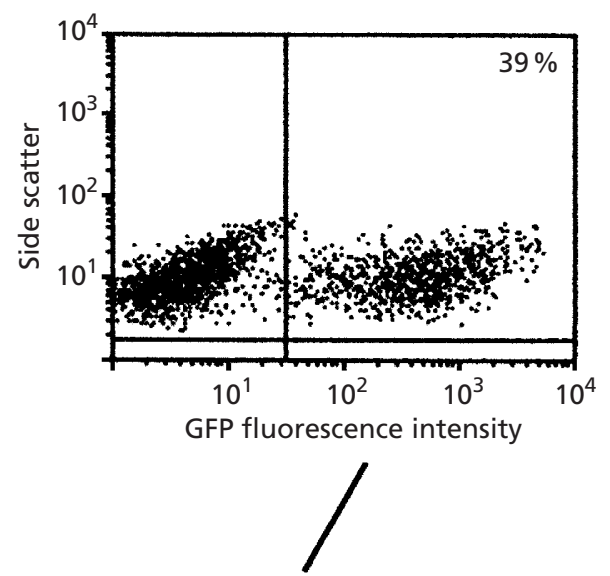

Sucrose counterselection of bacteria harbouring strong ex vivo-expressed promoters (d)

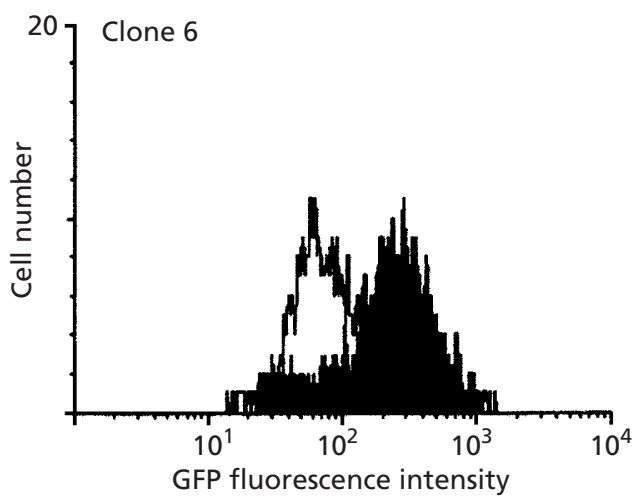

- Macrophage re-infection

- Selection of individual induced clones
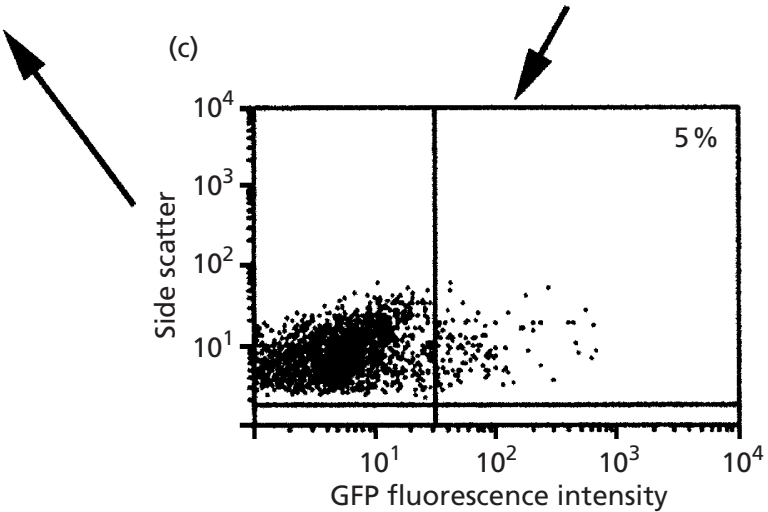

Fig. 3. Strategy for the isolation of $M$. tuberculosis promoter expressed within host cells. A library of random $M$. tuberculosis 103 DNA fragments $(0 \cdot 2-1.5 \mathrm{~kb})$ was constructed in the selection vector pJFX2 and transformed into M. bovis BCG. The proportion of bacteria displaying GFP fluorescence after growth in 7H9 culture medium was determined by FACS (a). Macrophages were infected with this pool for $6 \mathrm{~d}$, after which fluorescent cells were sorted, lysed and bacteria recovered in 7H9 medium. After three rounds of such in vivo enrichment a pool of rBCG displaying an augmented proportion of fluorescent bacteria was obtained (b). Bacteria in 7H9 medium were then incubated with sucrose $(0 \cdot 3 \%$, $6 \mathrm{~d})$ which resulted in the elimination of rBCG displaying intermediate to strong promoter activity outside the macrophage. (d) FACS histograms of macrophage-induced GFP-fluorescence of an isolated clone (clone 6). The relative fluorescence intensities of bacteria recovered from macrophages infected for $4 \mathrm{~h}$ (clear) or $6 \mathrm{~d}$ (filled) were compared.

detection of fluorescence in macrophages just after infection $(4 \mathrm{~h})$ to be a good measure of initial levels of $g f p$ expression as it accounts for any effect on fluorescence due to exposure to the culture medium and/or the preparation of bacteria from macrophages. As such, we calculated the level of intracellular induction as the GFP level at day 6 of infection compared to $4 \mathrm{~h}$ (termed day 0 ). Typically, the fluorescence level of clones grown in 7H9 culture medium was either the same or slightly lower than that observed for bacteria recovered from macrophages after the $4 \mathrm{~h}$ infection period (data not shown). Out of the approximately 400 individual rBCG clones examined, seven showed reproducibly greater levels of fluorescence after $6 \mathrm{~d}$ within the macrophage compared to GFP fluorescence at day 0 (Table 1). Three of these clones (1-3) were isolated from bacteria that did not undergo sucrose selection, while the remaining four (clones 4-7) were isolated by the combined fluorescence induction and sucrose counterselection procedures. A representative histogram of one of the isolated clones is shown in Fig. 3(d). Calculation of the increase in fluorescence demonstrated a range in intracellular induction of approximately two to threefold (Table 1).

\section{Identification of $M$. tuberculosis gene sequences under the control of macrophage-induced promoters}

The recent completion of the M. tuberculosis genome sequence permitted the identification and localization of the genes associated with promoters that exhibit differential expression intracellularly. The inserts of the seven clones were recovered by PCR and sequenced. The comparison with the M. tuberculosis genome is shown in Table 1. All sequences were located within the 
Table 1. Analyses of clones harbouring M. tuberculosis promoters displaying enhanced activity within macrophages

\begin{tabular}{|c|c|c|c|}
\hline Clone & $\begin{array}{l}\text { Insert size } \\
\quad(\mathbf{k b})\end{array}$ & $\begin{array}{l}\text { Induction in } \\
\text { macrophages } \\
\quad(- \text { fold })^{*}\end{array}$ & Gene/protein features \\
\hline 1 & $1 \cdot 4$ & $2 \cdot 07 \pm 0 \cdot 08$ & tkt gene encoding Rv1449c: transketolase of the pentose phosphate pathway \\
\hline 2 & $1 \cdot 4$ & $1 \cdot 98 \pm 0 \cdot 10$ & $\begin{array}{l}\text { fadB4 gene encoding Rv3141: strong similarity to quinone oxidoreductases and domains } \\
\text { in polyketide and fatty acid synthases }\end{array}$ \\
\hline 3 & $0 \cdot 4$ & $2 \cdot 22 \pm 0 \cdot 36$ & Gene encodes Rv3718c: function unknown \\
\hline 4 & $1 \cdot 2$ & $2 \cdot 23 \pm 0 \cdot 39$ & sse A gene encoding Rv3283: putative thiosulfate sulfurtransferase \\
\hline 5 & $1 \cdot 5$ & $1.93 \pm 0.05$ & cysD gene encoding Rv1285: probable sulfate adenylate transferase subunit 2 \\
\hline 6 & $1 \cdot 3$ & $3 \cdot 14 \pm 0 \cdot 21$ & $\begin{array}{l}\text { Gene encodes Rv0834c: member of the PE-PGRS subfamily of the M. tuberculosis } \\
\text { glycine/alanine-rich protein family }\end{array}$ \\
\hline 7 & $0 \cdot 4$ & $2 \cdot 22 \pm 0 \cdot 24$ & $\begin{array}{l}\text { Within Rv3206c; moeZ gene: encodes a probable MOE B-homologue, possibly involved } \\
\text { in cofactor synthesis }\end{array}$ \\
\hline
\end{tabular}

*Values for induction in macrophages (-fold) represent the fluorescence value of bacteria recovered from macrophages after $6 \mathrm{~d}$ of infection divided by the fluorescence level of the same bacteria recovered after $4 \mathrm{~h}$ of infection (day 0 ). Experiments were repeated at least three times and values represent the mean fluorescence induction level \pm SEM.

published genome sequence (Cole et al., 1998). For clones 1-6, sequences were located upstream of and reading into a postulated $M$. tuberculosis gene, strongly suggesting that the promoter corresponding to the identified genes was located within the isolated $M$. tuberculosis DNA fragments. The sequence of clone 7 was located within the gene encoding the M. tuberculosis Rv3206c (moeZ homologue) and thus may correspond to a cryptic promoter that demonstrates enhanced activity in vivo.

Of the seven clones, four showed strong similarity to previously described genes and coding sequences from a number of species (Table 1). Clone 1 corresponds to a gene exhibiting strong homology to transketolases of the pentose phosphate pathway. Clone 2 maps to the fadB4 gene, homologous to a 3-hydroxyl-CoA dehydrogenase, postulated to be involved in fatty acid metabolism. This gene is also strongly similar to quinone oxidoreductases, which serve a role as phase II detoxifying enzymes required for defence against the intake of xenobiotics. Clones 4 and 5 both correspond to genes possibly involved in the metabolism of sulphur. The sseA gene (clone 4) encodes a thiosulfate sulfurtransferase, which catalyses sulfur transfer from thiosulfate. The cysD gene (clone 5) encodes a sulfate adenylate transferase subunit $\mathrm{B}$, which is required for the activation of inorganic sulphate. Immediately downstream of $c y s D$ lies $c y s N$ which encodes the A subunit of the sulfate adenylate transferase complex. Clone 6 contains the postulated promoter region of a gene encoding an alanine/glycine-rich protein which is a member of the large PE-PGRS subfamily of M. tuberculosis (Cole et al., 1998).

\section{DISCUSSION}

A better understanding of the mechanisms utilized by M. tuberculosis to enhance its persistence within host cells would contribute greatly to the development of strategies to combat tuberculosis. As such intracellular persistence would depend on the coordinated regulation of intracellular gene expression by the bacterium, we have developed a genetic system which permits the isolation of $M$. tuberculosis promoters that display enhanced activity in vivo. Using a combination of the natural fluorescence of the A. victoria GFP and the counterselective properties of the B. subtilis SacB protein, we have identified seven promoters that demonstrate greater activity after prolonged residence within macrophages. At least four of these promoters corresponded to genes with homologues from other species (Table 1). Two of these gene products (CysD and SseA) are homologous to proteins involved in the utilization of sulphur and expression of the Chlamydomonas reinhardtii cysD homologue is enhanced when cells are exposed to sulphur-limiting conditions (Yildiz et al., 1996). Thus the observed up-regulation of $c y s D$ and sse $A$ could be due to a starvation-type response resulting from the limited availability of sulphur within the macrophage environment. The tkt gene (clone 1) is upregulated in the mouse cornea upon exposure to oxidative stress such as the presence of hydrogen peroxide (Salamon et al., 1998) and thus up-regulation of the M. tuberculosis homologue could be due to the stress response encountered by the bacterium during residence within the macrophage. Furthermore, the $t k t$ gene product has the ability to compensate for the loss of superoxide dismutase in Saccharomyces cerevisiae (Slekar et al., 1996), a protein which appears to play an important role in the cell's defence against toxic oxygen metabolites generated by phagocytic cells in an attempt to eliminate pathogenic invaders (Miller \& Britigan, 1997). The fact that the product of the fadB4 gene (clone 2) shares homology with quinone oxidoreductases, involved in the defence against xenobiotics, may again indicate an up-regulation of this gene in an attempt to counter defence mechanisms employed by the macrophage to eliminate M. tuberculosis during infection. In 
human epithelial cells, quinone oxidoreductase expression is induced by xenobiotics (Tumminia et al., 1993) and the gene has been shown to be under the control of anti-oxidant response elements (Itoh et al., 1997). Although no proposed function could be ascribed to the gene product associated with clone 6 , it corresponded to the large family of glycine-rich proteins of $M$. $t u$ berculosis, thus giving some insight into the possible role of at least one of this family which comprises a large portion of the organism's coding sequence (Cole et al., 1998).

The levels of intracellular induction obtained for the isolated promoters was reproducibly in the range of two to threefold. As GFP requires no substrate and thus no amplification step for the visualization of fluorescence, one could consider that an induction of threefold represents an actual threefold increase in expression, which would constitute a significant change in protein levels. Accordingly, most studies examining gene regulation from other mycobacteria have shown a similar level of induction. For example, 11 of 12 M. marinum promoters shown to be induced in vivo exhibited GFP induction levels of 1.4-3.4-fold; the one exception was a gene induced up to 15 -fold (Barker et al., 1998). In contrast to these results obtained with mycobacteria, studies with Salmonella using a similar mutant form of GFP showed in vivo induction typically in the range of 8-30-fold, with one promoter induced up to 443-fold (Valdivia \& Falkow, 1997). This difference may suggest a refining of host-cell-associated gene expression by $M$. tuberculosis such that small changes in expression are sufficient to exert the desired biological action, indicating an exquisite adaptation of the bacteria to its host. It is also possible that M. tuberculosis promoters strongly induced in vivo are present but were not identified in this study; for example, the M. tuberculosis acr gene appears to be induced around 100-fold within the murine macrophage, although this level was determined using the luciferase reporter gene and thus cannot accurately be compared to results obtained in this study using the GFP (Yuan et al., 1998).

This study selects for promoters that increase their level of expression within the macrophage and sustain such enhanced expression over a prolonged period of time (i.e. at least $6 \mathrm{~d}$ ). Thus we cannot exclude the fact that promoters switched on early and then down-regulated may have been eliminated by the use of our criteria. The GFP-SacB system could be modified accordingly to select for promoters with transient expression by changing the time of infection at which bacteria are analysed. It is also possible that by using a macrophage infection time of $4 \mathrm{~h}$ as our zero time point we could underestimate the level of induction if a promoter has already become active during this $4 \mathrm{~h}$ period. This does not seem to be the case for the promoters identified in this study, as levels of fluorescence in media and after infection for $4 \mathrm{~h}$ were essentially the same.

Our strategy to isolate promoters active in vivo made use of a novel system, where the properties of the $A$. victoria GFP and B. subtilis SacB protein were combined to enrich for the promoters of interest. Previously, the $\mathrm{SacB}$ protein has been shown to be an extremely powerful tool in the field of mycobacterial genetics. The ability to specifically eliminate $s a c B$-expressing mycobacteria in the presence of sucrose has been exploited to allow efficient allelic exchange and construction of transposon mutant libraries in both the slow- and fastgrowing mycobacteria (Pelicic et al., 1997). This is of particular importance in $M$. tuberculosis, where these techniques were always considered technically difficult and relatively inefficient. In all these studies, constitutive $\operatorname{sac} B$ expression was used at single sucrose concentrations to perform the counterselection. Our results demonstrate another use of this counterselective property of SacB, where recombinant bacteria could be selectively eliminated depending on their level of $s a c B$ expression. Such counterselection could be finely regulated depending on the concentration of sucrose used and the time of incubation. The demonstration that the level of $s a c B$ expression by mycobacteria corresponds to the level of killing may be exploited to study other facets of mycobacterial gene regulation; for example, this property was used to successfully isolate regulatory genes of Rhodobacter sphaeroides (Sabaty \& Kaplan, 1996).

Mycobacterial genes that undergo specific up-regulation within host cells may be important for the intracellular survival and virulence of the bacterium. Thus the deletion of such genes could provide M. tuberculosis strains limited in virulence, which may form the basis of attenuated vaccines to combat tuberculosis.

\section{ACKNOWLEDGEMENTS}

We thank Danielle Ensergueix for macrophage preparation, Jean Rauzier for DNA sequencing, Dr Philippe Métézeau and Hélène Kiefer for FACS sorting and Dr Brendan Cormack for provision of the pKEN-GFPmut2 vector. J.A.T. was the recipient of an Institut Pasteur Cantarini Fellowship. This work was supported by the European Community (grant BMH4 CT92167) and a Pasteur Merieux Connaught Research and Development grant.

\section{REFERENCES}

Barker, L. P., Brooks, D. M. \& Small, P. L. (1998). The identification of Mycobacterium marinum genes differentially expressed in macrophage phagosomes using promoter fusions to green fluorescent protein. Mol Microbiol 29, 1167-1177.

Clemens, D. L. (1996). Characterization of the Mycobacterium tuberculosis phagosome. Trends Microbiol 4, 113-118.

Cole, S. T., Brosch, R., Parkhill, J. \& 39 other authors (1998). Deciphering the biology of Mycobacterium tuberculosis from the complete genome sequence. Nature 393, 537-544.

Cormack, B. P., Valdivia, R. H. \& Falkow, S. (1996). FACSoptimized mutants of the green fluorescent protein (GFP). Gene 173, 33-38.

Dhandayuthapani, S., Via, L. E., Thomas, C. A., Horowitz, P. M., Deretic, D. \& Deretic, V. (1995). Green fluorescent protein as a marker for gene expression and cell biology of mycobacterial interactions with macrophages. Mol Microbiol 17, 901-912. 
Gercken, J., Pryjma, J., Ernst, M. \& Flad, H. D. (1994). Defective antigen presentation by Mycobacterium tuberculosis-infected monocytes. Infect Immun 62, 3472-3478.

Itoh, K., Chiba, T., Takahashi, S. \& 12 other authors (1997). An $\mathrm{Nrf2}$ /small Maf heterodimer mediates the induction of phase II detoxifying enzyme genes through antioxidant response elements. Biochem Biophys Res Commun 236, 313-322.

Kremer, L., Baulard, A., Estaquier, J., Poulain-Godefroy, O. \& Locht, C. (1995). Green fluorescent protein as a new expression marker in mycobacteria. Mol Microbiol 17, 913-922.

Miller, R. A. \& Britigan, B. E. (1997). Role of oxidants in microbial pathophysiology, Clin Microbiol Rev 10, 1-18.

Moors, M. A. \& Portnoy, D. A. (1995). Identification of bacterial genes that contribute to survival and growth in an intracellular environment. Trends Microbiol 3, 83-85.

Otal, I., Martin, C., Vincent-Levy-Frebault, V., Thierry, D. \& Gicquel, B. (1991). Restriction fragment length polymorphism analysis using IS6110 as an epidemiological marker in tuberculosis. J Clin Microbiol 29, 1252-1254.

Pelicic, V., Reyrat, J.-M. \& Gicquel, B. (1996a). Expression of the Bacillus subtilis sacB gene confers sucrose sensitivity on mycobacteria. J Bacteriol 178, 1197-1199.

Pelicic, V., Reyrat, J.-M. \& Gicquel, B. (1996b). Generation of unmarked directed mutations in mycobacteria using sucrose counterselectable suicide vectors. Mol Microbiol 20, 919-925.

Pelicic, V., Jackson, M., Reyrat, J.-M., Jacobs, W. R., Jr, Gicquel, B. \& Guilhot, C. (1997). Efficient allelic exchange and transposon mutagenesis in Mycobacterium tuberculosis. Proc Natl Acad Sci USA 94, 10955-10960.

Quandt, J. \& Hynes, M. F. (1993). Versatile suicide vectors which allow direct selection for gene replacement in gram-negative bacteria. J Bacteriol 127, 15-21.

Ranes, M. G., Rauzier, J., Lagranderie, M., Gheroghiu, M. \& Gicquel, B. (1990). Functional analysis of pAL5000, a plasmid from Mycobacterium fortuitum: construction of a 'Mini' mycobacterium-Escherichia coli shuttle vector. J Bacteriol 172, 27932797.
Raviglione, M. C., Snider, D. E. \& Kochi, A. (1995). Global epidemiology of tuberculosis. Morbidity and mortality of a worldwide epidemic. JAMA 273, 220-226.

Sabaty, M. \& Kaplan, S. (1996). $M g p S$, a complex regulatory locus involved in the transcriptional control of the $p u c$ and $p u f$ operons in Rhodobacter sphaeroides. J Bacteriol 178, 35-45.

Salamon, C., Chervenak, M., Piatigorsky, J. \& Sax, C. M. (1998). The mouse transketolase (TKT) gene: cloning, characterization and functional promoter analysis. Genomics 48, 209-220.

Slekar, K. H., Kosman, D. J. \& Culotta, V. C. (1996). The yeast copper/zinc superoxide dismutase and the pentose phosphate pathway play overlapping roles in oxidative stress protection. $J$ Biol Chem 271, 28831-28836.

Timm, J., Perilli, M. G., Duez, C. \& 9 other authors (1994). Transcription and expression analysis, using $l a c Z$ and $p h o A$ gene fusions, of Mycobacterium fortuitum $\beta$-lactamase genes cloned from a natural isolate and a high-level $\beta$-lactamase producer. $\mathrm{Mol}$ Microbiol 12, 491-504.

Tumminia, S. J., Rao, P. V., Zigler, J. S., Jr \& Russell, P. (1993). Xenobiotic induction of quinone oxidoreductase activity in lens epithelial cells. Biochim Biophys Acta 1203, 251-259.

Valdivia, R. H. \& Falkow, S. (1996). Bacterial genetics by flow cytometry: rapid isolation of Salmonella typhimurium acidinducible promoters by differential fluorescence induction. Mol Microbiol 22, 367-378.

Valdivia, R. H. \& Falkow, S. (1997). Fluorescence-based isolation of bacterial genes expressed within host-cells. Science 277, 2007-2011.

Yildiz, F. H., Davies, J. P. \& Grossman, A. (1996). Sulfur availability and the SAC1 gene control adenosine triphosphate sulfurylase gene expression in Chlamydomonas reinhardtii. Plant Physiol 112, 669-675.

Yuan, Y., Crane, D. G., Simpson, R. M., Zhu, Y. Q., Hickey, M. J., Sherman, D. R. \& Barry, C. E. (1998). The $16-\mathrm{kDa} \alpha$-crystallin (Acr) protein of Mycobacterium tuberculosis is required for growth in macrophages. Proc Natl Acad Sci USA 95, 9578-9583.

Received 28 April 1999; revised 21 June 1999; accepted 25 June 1999. 